\title{
Reviewer Acknowledgements for Global Journal of Health Science, Vol. 9, No. 6
}

Global Journal of Health Science wishes to acknowledge the following individuals for their assistance with peer review of manuscripts for this issue. Their help and contributions in maintaining the quality of the journal are greatly appreciated.

Global Journal of Health Science is recruiting reviewers for the journal. If you are interested in becoming a reviewer, we welcome you to join us. Please find the application form and details at http://recruitment.ccsenet.org and e-mail the completed application form to gjhs@ccsenet.org.

\section{Reviewers for Volume 9, Number 6}

Abiodun Adeniran
Agnes N. Anarado
Alexander Domnich
Alvisa Palese
Ama P. Fenny
António Calha
Budi Kusnoto
Cornelis Justien
Cuneyt Narin
David Lindsay
Dilek Pirim
Kartheek R Balapala
Evanthia Sakellari
Francesca Maria Pernice-Duca
Gabriele Messina
Gretel Gonzalez Colmenares
Guihong Cai
Hadii M Mamudu
Horng-Jyh Tsai
Hülya Yardimci
Isac Silva De Jesus
Jan Chrastina
Jean Ann Landry
Jiunn-Woei Lian
Jo Ann M. Bamdas
Kartheek Balapala
Ana

Abiodun Adeniran

Ken $\mathrm{Gu}$

Madhu Ouseph

Manuela Mauceri

Marcel Wullschleger

Maria Malliarou

Mark Tully

Meng Zhao

Nant Thin Thin Hmwe

Nazisa Hejazi

Nhathai Phan

Nishant Negi

Pi-Ming Yeh

Giancarlo Fortino

Raildo Coqueiro

Regina E.Ella

Robert Sloan

Roger Ho

Rongfang $\mathrm{Hu}$

Ru-Jeng Teng

Sandra J. Waters

Sarandeep Boyanapalli

Satoshi Horiuchi

Shwn-Meei Lee

Toby Raeburn

Vasundhara

Yasam Kemal Akpak 